\title{
Blendas PVC/NBR por Processamento Reativo II: Caracterização Físico-Mecânica e Morfológica
}

\author{
Fábio R. Passador, Luiz A. Pessan \\ Departamento de Engenharia de Materiais, UFSCar \\ Antonio Rodolfo Jr. \\ Braskem S/A
}

\begin{abstract}
Resumo: Vulcanização dinâmica é o processo de vulcanização de um elastômero durante a mistura no estado fundido com um termoplástico. Por este processo, o elastômero adquire resistência mecânica através do aumento do módulo de elasticidade, dureza, resistência à fadiga e abrasão. Neste trabalho, buscou-se avaliar as propriedades físico-mecânicas e as características morfológicas de blendas poliméricas constituídas de Poli(cloreto de Vinila) e borracha nitrílica, PVC/NBR, obtidas por processamento reativo. A vulcanização dinâmica melhorou o desempenho mecânico destas blendas, notadamente pelo aumento da rigidez desses sistemas, sendo obtido um aumento de $205 \%$ no módulo elástico de blendas vulcanizadas com $10 \%$ em massa de NBR em comparação com blendas convencionais. Através de análises de MEV, revelou-se uma morfologia bifásica, sendo que a formação de ligações cruzadas deve ocorrer preferencialmente no interior das partículas do elastômero, contribuindo para o aumento na resistência mecânica final das blendas obtidas por processamento reativo.
\end{abstract}

Palavras-chave: Vulcanização dinâmica, poli (cloreto de vinila), borracha nitrílica, blenda PVC/NBR, propriedades físicomecânicas.

\section{PVC/NBR Blends by Reactive Processing II: Physical-Mechanical and Morphological Characterization}

Abstract: Dynamic vulcanization is a process of vulcanization of an elastomer during melt mixing with a thermoplastic. This process increases the mechanical resistance of elastomers through the increase of elastic modulus, hardness and abrasion/ fatigue resistance. In this study, it was evaluated the physical-mechanical and morphological behaviors of the PVC/NBR blends obtained by reactive processing. The dynamic vulcanized blends have a better performance compared to the similar conventional ones. It was observed an increase of $205 \%$ in the elastic modulus to the dynamic vulcanized blend PVC/NBR (90/10) compared with the conventional blends. The morphology of the blends examined by scanning electron microscopy evidenced the crosslinking formation only in the elastomeric phase. The vulcanized rubber particles are responsible by the increase of stiffness and consequently displayed better mechanical properties.

Keywords: Dynamic vulcanization, poly (vinyl chloride), nitrile rubber, reactive processing, PVC/NBR blends, physicalmechanical properties.

\section{Introdução}

O crescente avanço tecnológico gera a necessidade de desenvolver novos materiais que apresentem características específicas. O desenvolvimento de blendas PVC/NBR de elevado desempenho, para aplicações tecnológicas específicas, vem sendo motivo de estudos de vários grupos de pesquisa ${ }^{[1-4]}$. Dentre os principais destaca-se o processo de vulcanização do elastômero durante a mistura no estado fundido com um termoplástico ${ }^{[5,6]}$. A vulcanização in situ da fase elastomérica durante o processamento das blendas PVC/NBR visa a obtenção de produtos com boa resistência química e mecânica em altas temperaturas, para aplicações como, por exemplo, mangueiras de alto desempenho na indústria automotiva.

As principais vantagens do processo de vulcanização de elastômeros durante mistura com termoplástico consistem na formação de uma rede molecular tridimensional reticulada no elastômero que permite manter ou incrementar o comportamento elástico borrachoso, diminuindo o escoamento plástico. O elastômero se torna insolúvel, mais resistente fisicamente num intervalo de temperatura maior que o da borracha não vulcanizada e adquire resistência mecânica através do aumento do módulo de elasticidade, dureza, resistência à fadiga e abrasão $0^{[6-8]}$.

A morfologia de blendas obtidas através de vulcanização dinâmica apresenta características bastante particulares. A Figura 1 apresenta um esquema da morfologia da blenda PP/EPDM obtida por vulcanização dinâmica onde as partículas elastoméricas estão dispersas na matriz termoplástica e ocorre a formação de ligações cruzadas apenas no interior da fase elastomérica ${ }^{[9]}$. Porém há poucas informações sobre a morfologia de blendas PVC/NBR obtidas por processa-

Autor para correspondência: Fábio R. Passador, Departamento de Engenharia de Materiais, UFSCar, Via Washington Luís, km 235, Caixa Postal 676, CEP 13565-905, São Carlos, SP, Brasil.E-mail: fpassador@polvo.ufscar.br. 


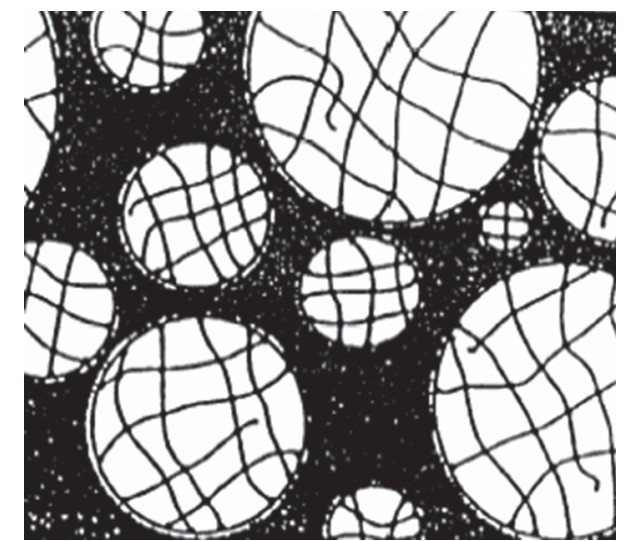

Figura 1. Esquema da morfologia de formação de ligações cruzadas na blenda PP/EPDM obtida por vulcanização dinâmica ${ }^{[9]}$.

mento reativo, assim como sobre as propriedades físico-mecânicas destas blendas.

A proposta desse trabalho foi avaliar as propriedades físico-mecânicas e a morfologia de blendas PVC/NBR obtidas por processamento reativo e comparar os resultados com blendas PVC/NBR convencionais. Dessa forma, foram preparadas blendas PVC/NBR nas proporções 90/10, 80/20 e 70/30\% em massa através de processamento reativo em reômetro de torque e para promover a formação de ligação cruzada in situ da NBR utilizou-se um sistema de vulcanização à base de enxofre e combinação de aceleradores (2,2ditiomercaptobenzotiazol (MBTS) e dissulfeto de tetrametiltiuram (TMTD)). As blendas foram caracterizadas quanto às propriedades mecânicas por ensaios de tração, rasgo, dureza e deformação permanente à compressão e a análise morfológica foi feita por microscopia eletrônica de varredura.

\section{Experimental}

\section{Materiais e preparação das blendas}

Os materiais utilizados e a preparação das blendas PVC/ NBR convencionais e vulcanizadas in situ nas proporções 90/10, 80/20 e 70/30\% em massa estão descritos no artigo anterior ${ }^{[10]}$.

\section{Ensaios físico-mecânicos}

A caracterização física foi feita através da determinação da massa específica utilizando um gravitômetro da Fisher Young Gravitometer, segundo a norma ASTM D-792, em triplicata.

A caracterização mecânica foi realizada a partir dos ensaios de tração, resistência ao rasgo e dureza das blendas.

Os ensaios de tração foram realizados numa máquina universal de ensaios MTS modelo Aliance RT/5 seguindo a norma ASTM D-638, em corpos de prova do tipo IV com formato de "gravata". Foi utilizado uma célula de carga de $5 \mathrm{KN}$, velocidade da travessa de $500 \mathrm{~mm} / \mathrm{min}$, com a utilização de extensômetro.
Os ensaios de resistência ao rasgo foram realizados numa máquina universal de ensaios MTS modelo Aliance RT/5 seguindo a norma ASTM D-1004, com velocidade da travessa de $51 \mathrm{~mm} / \mathrm{min}$.

Para o ensaio de dureza Shore A foi utilizado um durômetro com relógio digital da Bareiss, segundo a norma ASTM D-2240. Foram preparados corpos de prova cilíndricos de $6,0 \pm 0,2 \mathrm{~mm}$ de espessura e 5,0 $\pm 0,2 \mathrm{~cm}$ de diâmetro. Foram realizadas cinco medidas em cada amostra.

A determinação da deformação permanente à compressão foi realizada de acordo com a norma ASTM D-395 método B. Foram preparados corpos de prova no formato cilíndrico de 6,0 $\pm 0,2 \mathrm{~mm}$ de espessura e 13,0 $\pm 0,2 \mathrm{~mm}$ de diâmetro. As amostras foram comprimidas a $25 \%$ de sua altura inicial durante 2 horas e após este tempo o dispositivo com a amostra foi colocado em estufa a $100{ }^{\circ} \mathrm{C}$ por 22 horas. Os testes foram realizados em duplicata.

\section{Caracterização morfológica}

A morfologia foi analisada utilizando um microscópio eletrônico de varredura (MEV) da marca Philips, modelo XL30 FEG. A superfície das amostras foi atacada quimica-

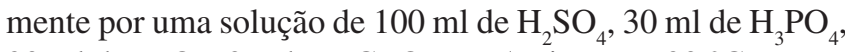
$30 \mathrm{ml} \mathrm{de} \mathrm{H}_{2} \mathrm{O}$ e $3 \mathrm{~g}$ de $\mathrm{K}_{2} \mathrm{Cr}_{2} \mathrm{O}_{7}$ por 5 minutos a $30^{\circ} \mathrm{C}$ e recobertas com ouro para a observação em microscópio.

\section{Resultados e Discussão}

\section{Avaliação das propriedades físico-mecânicas das blendas}

A massa específica é um parâmetro importante na aplicação das blendas, uma vez que determina a densidade do produto final. A Tabela 1 apresenta os resultados de massa específica do composto de PVC, da NBR e das blendas convencionais e vulcanizadas obtidas neste trabalho.

De maneira geral, observou-se que o aumento do teor de elastômero reduz progressivamente a massa específica das blendas, tal como esperado quando se incorpora uma fase com menor massa específica que a matriz. Em todos os casos, entretanto, verifica-se que a incorporação de teores crescentes de elastômero não altera de forma significativa a massa

Tabela 1. Massa específica do composto de PVC, da NBR, NBR vulcanizada (NBRv) e das blendas convencionais (BC) e vulcanizadas (BV) com teores de 10, 20 e $30 \%$ em massa de NBR.

\begin{tabular}{lc}
\hline \multicolumn{1}{c}{ Amostra } & Massa específica $\left(\mathbf{g} / \mathbf{c m}^{\mathbf{3}}\right)$ \\
\hline Composto de PVC & $1,23 \pm 0,01$ \\
NBR & $1,02 \pm 0,02$ \\
NBRv & $1,06 \pm 0,03$ \\
BC 10 & $1,19 \pm 0,01$ \\
BV 10 & $1,19 \pm 0,01$ \\
BC 20 & $1,17 \pm 0,01$ \\
BV 20 & $1,17 \pm 0,01$ \\
BC 30 & $1,15 \pm 0,01$ \\
BV 30 & $1,15 \pm 0,01$ \\
\hline
\end{tabular}


específica das blendas tanto convencionais quanto vulcanizadas in situ, ou seja, a adição do sistema de cura para formação de ligações cruzadas não influenciou este parâmetro, sendo os valores obtidos próximos aos valores para as blendas convencionais.

O composto de $\mathrm{PVC}$, as blendas $\mathrm{PVC} / \mathrm{NBR}$ convencionais e vulcanizadas e as borrachas nitrílicas foram caracterizadas quanto suas resistências mecânicas através de ensaios de tração, rasgo, dureza e deformação permanente à compressão.

A partir do ensaio de tração foram obtidas informações sobre a tensão na ruptura $\left(\sigma_{\mathrm{r}}\right)$, deformação na ruptura $\left(\varepsilon_{\mathrm{r}}\right)$ e módulo elástico (E) dos sistemas estudados, que estão apresentados na Tabela 2.

Analisando os resultados de tensão na ruptura observouse que a NBR pura possui baixa tensão na ruptura e que o processo de vulcanização (NBRv) promove aumento no valor de tensão de ruptura e consequentemente na resistência mecânica da NBR ${ }^{[9]}$. Variando-se o teor de NBR nas composições das blendas convencionais (BC) estudadas, pode-se notar que todas as blendas apresentaram valores superiores de tensão na ruptura comparados à NBR. Pode-se observar ainda, que o valor da tensão na ruptura diminui à medida que aumenta o teor de NBR não vulcanizada em comparação ao valor do composto de PVC. A blenda BC 10 apresentou valor próximo de tensão na ruptura ao composto de PVC. No caso das blendas obtidas por processamento reativo (BV) observou-se que todas as composições obtiveram valores superiores de tensão na ruptura comparados às blendas convencionais. Aumentando-se o teor de NBR vulcanizada na composição observou-se uma diminuição nos valores de tensão de ruptura. O processo de vulcanização tornou a fase NBR mais rígida o que contribuiu para o aumento na resistência mecânica das blendas ${ }^{[11]}$.

No que concerne a deformação na ruptura da borracha nitrílica pura (NBR) e vulcanizada (NBRv) pode-se observar que a formação de ligações cruzadas diminui consideravelmente a deformação, uma vez que diminui a elasticidade do elastômero, tornando-o mais rígido. O composto de PVC estudado possui grande deformação na ruptura devido à grande quantidade de plastificante utilizada em sua formu-

Tabela 2. Tensão na ruptura $(\sigma)$, deformação na ruptura $(\varepsilon)$ e módulo elástico (E) do composto de PVC, das borrachas nitrílicas e das blendas convencionais (BC) e vulcanizadas (BV) com teores de 10, 20 e 30\% em massa de NBR.

\begin{tabular}{lccc}
\hline \multicolumn{1}{c}{ Amostra } & $\sigma_{\mathrm{r}}(\mathbf{M P a})$ & $\varepsilon_{\mathrm{r}}(\%)$ & $\mathbf{E}(\mathbf{M P a})$ \\
\hline Composto de PVC & $15,2 \pm 0,2$ & $411,6 \pm 11,6$ & $10,55 \pm 0,16$ \\
NBR & $0,9 \pm 0,3$ & $594,6 \pm 15,7$ & $1,36 \pm 0,03$ \\
NBRv & $2,6 \pm 0,2$ & $147,3 \pm 9,9$ & $2,52 \pm 0,07$ \\
BC 10 & $14,7 \pm 0,2$ & $799,1 \pm 19,6$ & $2,61 \pm 0,26$ \\
BV 10 & $15,1 \pm 0,2$ & $394,1 \pm 11,8$ & $7,95 \pm 0,11$ \\
BC 20 & $12,5 \pm 0,3$ & $382,2 \pm 12,6$ & $6,29 \pm 0,09$ \\
BV 20 & $13,2 \pm 0,3$ & $136,3 \pm 10,6$ & $11,21 \pm 0,11$ \\
BC 30 & $11,1 \pm 0,3$ & $365,2 \pm 11,5$ & $5,17 \pm 0,13$ \\
BV 30 & $13,2 \pm 0,6$ & $311,3 \pm 3,6$ & $5,45 \pm 0,11$ \\
\hline
\end{tabular}

lação, que auxilia no aumento da flexibilidade das cadeias poliméricas ${ }^{[12]}$. Em todas as blendas estudadas observou-se que àquelas obtidas por processamento reativo obtiveram menores valores de deformação na ruptura, devido à formação de reticulados nas cadeias do elastômero e conseqüente aumento da densidade de ligações cruzadas.

O módulo de elasticidade ou módulo de Young é medido pela razão entre a tensão e a deformação, dentro do limite elástico, em que a deformação é totalmente reversível e proporcional à tensão, estando relacionado à rigidez do material ${ }^{[13,14]}$. Elastômeros possuem módulos de elasticidade relativamente baixos, e o processo de vulcanização aumenta a rigidez das cadeias elastoméricas devido a formação de ligações cruzadas contribuindo para o aumento o valor do módulo elástico ${ }^{[7]}$. Analisando os resultados da Tabela 2 pode-se observar o aumento no módulo elástico da NBR vulcanizada (NBRv) em comparação a NBR pura. Aumentando-se o teor de NBR na composição das blendas convencionais verificase a diminuição do módulo elástico em comparação com o valor obtido para o composto de PVC. Observou-se em todos os casos que o processo de vulcanização aumentou o módulo elástico das blendas vulcanizadas, devido ao maior grau de reticulação que proporcionou aumento na rigidez da fase elastomérica.

De maneira geral, os resultados obtidos através do ensaio de tração para as blendas vulcanizadas mostraram que com a utilização do sistema de cura baseado na mistura de aceleradores e enxofre é possível obter blendas por processamento reativo com desempenho mecânico superior às blendas convencionais. Sendo obtido um aumento de $205 \%$ no módulo elástico de blendas vulcanizadas com 10\% em massa de NBR em comparação com a blenda convencional, com mesmo teor de NBR.

A Figura 2 apresenta os resultados do ensaio de resistência ao rasgo do composto de PVC, da NBR e das blendas convencionais e vulcanizadas estudadas.

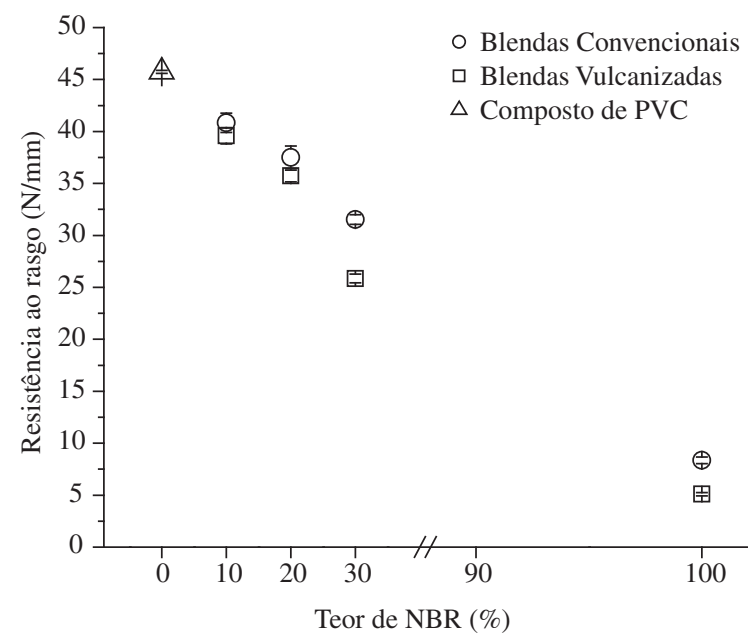

Figura 2. Resistência ao rasgo (N/mm) em função do teor de NBR do composto de PVC, da NBR e das blendas convencionais e vulcanizadas in situ. 
O processo de vulcanização aumenta a resistência ao rasgo devido à formação de reticulados nas cadeias poliméricas, como observado para NBRv em comparação a NBR pura. Analisando os resultados da Figura 2 observou-se que o aumento do teor de NBR diminui a resistência ao rasgo nas blendas convencionais devido à baixa resistência ao rasgo da NBR pura, quando comparado ao valor obtido para o composto de PVC. No caso das blendas obtidas por processamento reativo, observou-se aumento na resistência ao rasgo de todas as composições comparado às blendas convencionais. A maior resistência ao rasgo das blendas obtidas por processamento reativo está diretamente relacionada à densidade de ligações cruzadas, porém é preciso considerar outros fatores que são importantes na obtenção destas blendas, como a distribuição e o tamanho da fase dispersa formada durante o processamento que pode influenciar diretamente nos mecanismos de ruptura das cadeias e, consequentemente, no desempenho mecânico das blendas.

A Figura 3 apresenta os resultados de dureza Shore A do composto de PVC, da NBR e das blendas convencionais e vulcanizadas.

O composto de PVC apresentou dureza superior a NBR e NBRv. Para as blendas convencionais, observou-se que o aumento no teor de borracha nitrílica na composição das blendas diminui a dureza dos compostos, em comparação à dureza do composto de PVC, devido à baixa dureza da borracha nitrílica pura. No caso das blendas vulcanizadas, observou-se que o processo de vulcanização influenciou pouco neste parâmetro obtendo-se valores próximos de dureza para as blendas convencionais e vulcanizadas in situ.

A Figura 4 apresenta os resultados de deformação permanente à compressão do composto de $\mathrm{PVC}$, da NBR e das blendas convencionais e vulcanizadas in situ.

A maior resistência à compressão está expressa em termos da menor deformação permanente à compressão (DCP). Dessa forma, pode-se observar que NBR pura possui boa resistência à compressão. Observou-se que o valor obtido pelo vulcanizado (NBRv) é maior que para a borracha nitrílica pura, uma vez que o processo de formação de ligações cruza-

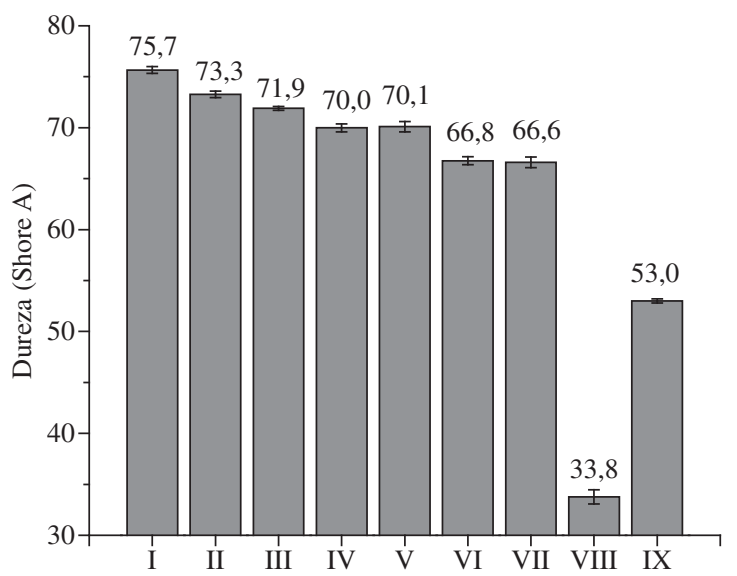

Figura 3. Dureza Shore A: (I) Composto de PVC; (II) BC 10; (III) BV 10; (IV) BC 20; (V) BV 20; (VI) BC 30; (VII) BV 30; (VIII) NBR; e (IX) NBRv.

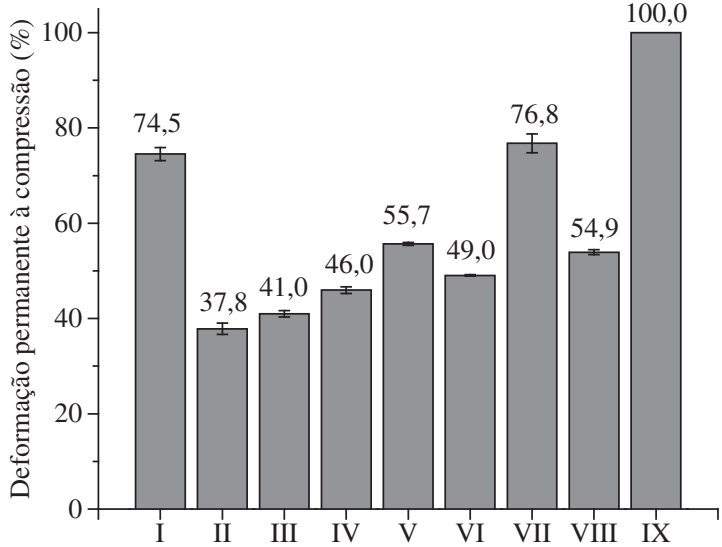

Figura 4. Deformação permanente à compressão (\%): (I) Composto de PVC; (II) BC 10; (III) BV 10; (IV) BC 20; (V) BV 20; (VI) BC 30; (VII) BV 30; (VIII) NBR; e (IX) NBRv.

das diminui as propriedades elásticas do elastômero tornando-o menos resistente à compressã $0^{[7,15]}$.

No caso das blendas convencionais observou-se aumento da resistência à compressão em comparação a NBR pura, enquanto que no caso das blendas vulcanizadas in situ, observou-se que aumentando o teor de NBR ocorre uma diminuição da resistência à compressão, que é devido ao maior grau de reticulação destas blendas. A resistência à compressão assim como outras propriedades mecânicas não é função apenas da densidade de ligações cruzadas, mas também do tipo de ligação formada. A característica deste sistema, em particular, é a formação de ligações mono e dissulfídicas, onde a deformação do vulcanizado é reduzida.

\section{Avaliação morfológica das blendas PVC/NBR obtidas por processamento reativo}

A Figura 5 apresenta as morfologias obtidas por MEV das blendas PVC/NBR vulcanizadas in situ.

$\mathrm{O}$ ataque químico utilizado para extração da fase elastomérica não foi eficaz, uma vez que o processo de vulcanização tornou o elastômero mais resistente quimicamente, porém observou-se que este ataque provocou um aparente inchamento da fase elastomérica sendo possível observar o tamanho e a dispersão desta fase. Analisando as micrografias, considerando as características do sistema de cura empregado e as citações da literatura $^{[5,7,9]}$, pode-se considerar que a formação de ligações cruzadas ocorreu apenas no interior da fase elastomérica. Analisando a Figura 5 pode-se observar que aumentando o teor de NBR nas blendas PVC/NBR, ocorreu aumento da quantidade de fase dispersa. A blenda BV 10 (Figura 5a) apresentou heterogeneidade no tamanho da fase elastomérica sendo possível observar a formação de alguns aglomerados.

As blendas obtidas por processamento reativo apresentaram morfologia bifásica com tamanho reduzido de partículas de elastômero. Este fato pode ser um dos responsáveis pelo melhor desempenho mecânico obtido para as blendas em que ocorreu o processo de formação de ligações cruzadas in situ 


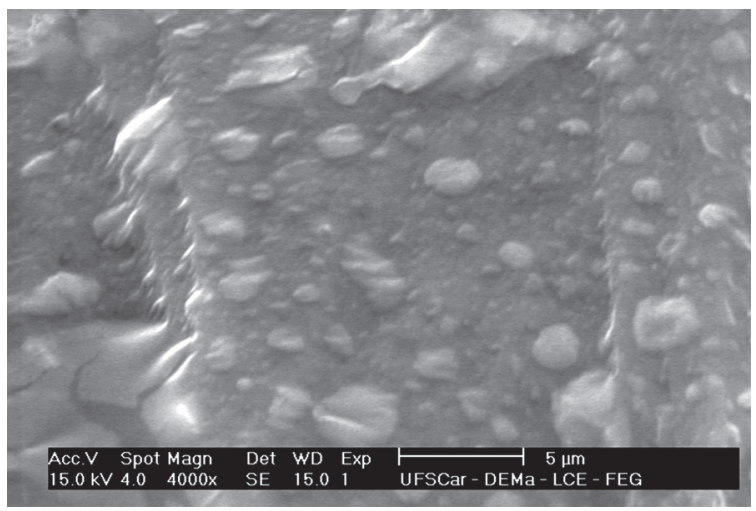

(a)

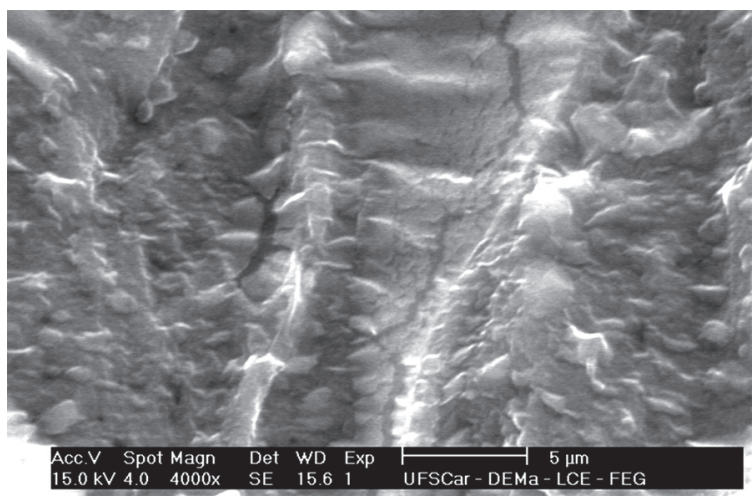

(b)

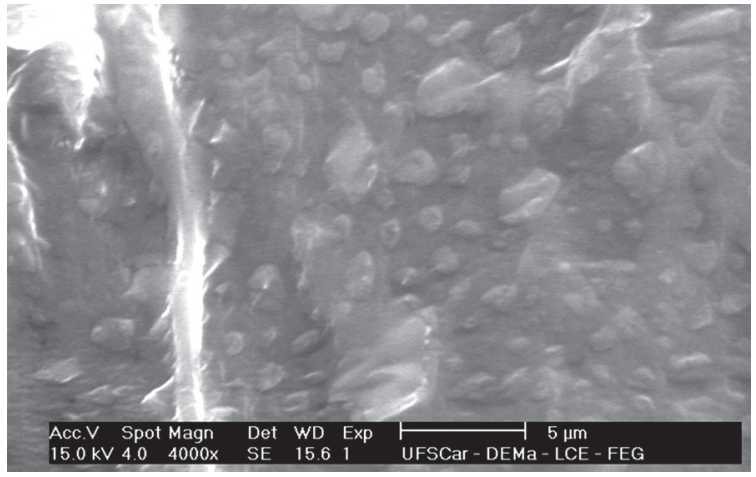

(c)

Figura 5. Micrografias de $\mathrm{MEV}$ das blendas $\mathrm{PVC} / \mathrm{NBR}$ vulcanizadas a) BV 10; b) BV 20; e c) BV 30. Aumento de 4000x.

da borracha nitrílica em comparação com sistemas convencionais.

\section{Conclusões}

No desenvolvimento deste trabalho foi possível comprovar que o processamento reativo da blenda PVC/NBR com formação de ligações cruzadas in situ entre as cadeias de NBR melhora o desempenho mecânico destas blendas, notadamente pelo aumento da rigidez desses sistemas, sendo obtido um aumento de $205 \%$ no módulo elástico de blendas vulcanizadas com $10 \%$ em massa de NBR em comparação com blendas convencionais.
O processo de vulcanização aumenta a resistência ao rasgo das blendas PVC/NBR obtidas devido à formação de reticulados nas cadeias poliméricas, porém ocorre uma diminuição na resistência à compressão destes sistemas.

Através das análises de MEV, revelou-se uma morfologia bifásica para as blendas PVC/NBR, sendo o PVC a matriz e a NBR a fase dispersa e pode-se considerar que a formação de ligações cruzadas ocorreu no interior das partículas do elastômero, contribuindo para o aumento na resistência mecânica final das blendas obtidas por processamento reativo.

\section{Agradecimentos}

Os autores agradecem à CAPES pelo apoio financeiro e à Braskem S.A. através do programa NEO PVC (Núcleo de Estudos Orientados do PVC).

\section{Referências Bibliográficas}

1. Manoj N. R.; De P. P.; De S. K. - J. Appl. Polym. Sci., 49, p.133 (1993).

2. Manoj N. R.; De P. P. - Polym., 39, p.733 (1998).

3. Gosh P.; Sem A. K., Ray P. - Polym., 33, p.744 (1992).

4. Oravec J.; Oishi Y.; Hirahara H.; Mori K. - Polym. Inter., 32, p.303 (1993).

5. Coran A. Y. in "Handbook of elastomers - new developments and technology", Stephens H. L., Ed.; Dekker Inc, New York (1988).

6. Walker B. M.; Rader C. P. - "Handbook of thermoplastic elastomers", Van Nostrand Reinhold, New York (1988).

7. Hills D. A. - "Heat Transfer and Vulcanisation of Rubber", Applied Science Publishers Ltd., London (1971).

8. Browmick A. K.; Hall M. M.; Benarey H. A. - "Rubber products manufacturing technology", Marcel Dekker Inc, New York (1997).

9. Akiba M.; Hashim A. S. - Prog. Polym. Sci., 22, p.475 (1997).

10. Passador F. R.; Pessan L. A.; Rodolfo Jr A. - Pol.: Ciênc. Tecnol., 17, p.80 (2007).

11. Oliveira M. G.; Soares B. G. - Pol.: Ciênc.Tecnol., 12, p.11 (2002).

12. Rodolfo Jr., A.; Nunes, L. R.; Ormanji, W. - "Tecnologia do PVC". Proeditores/Braskem, São Paulo (2006).

13. Ismail, H.; Tan, S.; Poh, B. T. - J. Elast. Plast., 33, p. 251 (2001).

14. Mousa, A.; Ishiaku, U. S.; Ishak, Z. A. M. - Polym. Bulletin, 53, p. 203 (2005).

15. Ehabe, E. E.; Farid, S. A. - Europ. Polym. J., 37, p. 329 (2001). 